\title{
Experimental strategies for avoiding saturation effects in ESI-MS
}

\author{
Alan An Jung Wei, Anuj Joshi, Yuxuan Chen and J. Scott Mclndoe* \\ Department of Chemistry, University of Victoria, PO Box 1700 STN CSC, Victoria, BC V8W 2Y2, \\ Canada. \\ Fax: +1 (250) 721-7147; Tel: +1 (250) 721-7181; E-mail: mcindoe@uvic.ca
}

\begin{abstract}
All instruments with detectors are prone to saturation effects at high concentration, and mass spectrometers are no exception. The very high sensitivity of mass spectrometry makes the onset of saturation occur at lower concentrations than other methods, and in cases where the analyte of interest is very reactive, concentrations at which saturation can be problematic may be necessary in order to ensure decomposition is mitigated. Indications that saturation is occurring are provided, and some data processing strategies are outlined, followed by a range of detuning strategies that may be employed to reduce saturation effects in the context of electrospray ionization mass spectrometry (ESI-MS), including lowering voltages on detector or capillary, increasing cone gas flow rates, or adjusting the probe position. A combination of strategies generally allows researchers to make the best possible compromises when studying compounds at relatively high concentration.
\end{abstract}

\section{Graphical abstract}

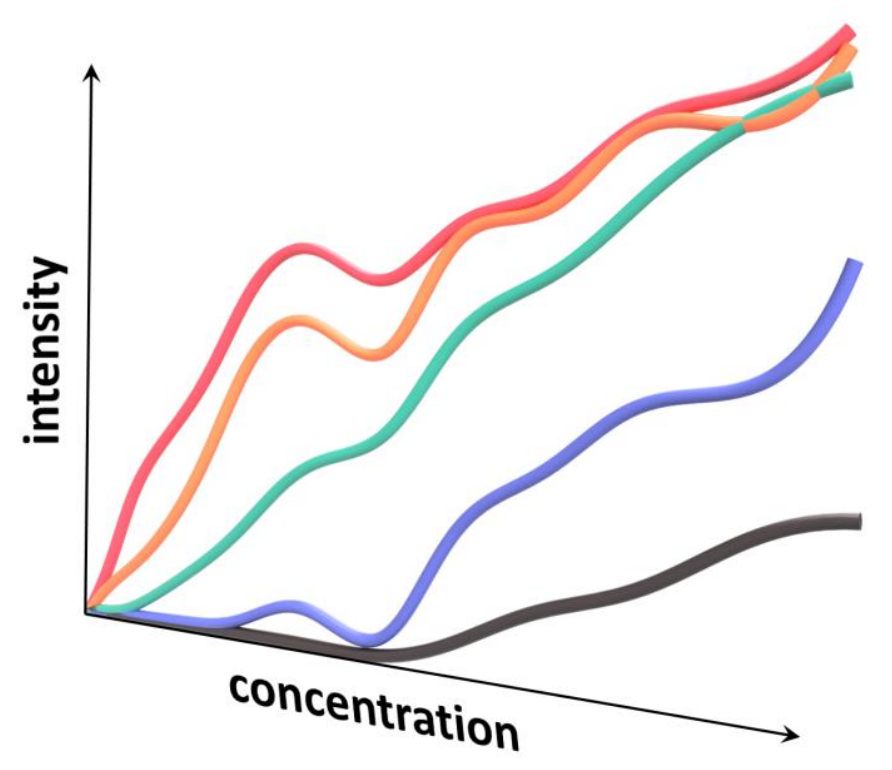




\section{Introduction}

Mass spectrometrists are tireless in their search for sensitivity [1]. Instrumentation and methodologies are in constant development in order to push the limits of detection lower and the linear dynamic range wider, and astonishing feats of characterization have been achieved as a result. For most chemists, and particularly those hunting trace analytes or dealing with incredibly complicated mixtures of biological origin, this quest is an entirely desirable one. However, a few of us use mass spectrometry to monitor the reactions of highly reactive compounds [2][3][4], and the raw sensitivity of the technique can prove to be a double-edged sword. Highly reactive compounds will interact rapidly and deleteriously with both oxygen and moisture [5][6][7], which are difficult to remove from solvents beyond $5 \mathrm{ppm}$. Yet mass spectrometers can effortlessly characterize charged species at this level, and far below. So we have a conundrum: on one hand, we want to run the analysis at as high a concentration as possible, because dilution will not only greatly slow the rate at which the reaction we're interested in will occur, it will also introduce relatively large quantities of undesirable compounds that can cause analyte decomposition. On the other hand, high concentrations are problematic for mass spectrometric analysis [8], causing issues of ion suppression [9] and saturation.

The main obstacle caused by saturation is the inability to acquire an accurate quantification of species [10], as the spectra no longer reflects the "real" concentration of the sample. There are a few possibilities that explain the phenomenon of saturation due to a higher concentration. Aside from previously mentioned ion suppression, the finite amount of excess charge [11] and the limited space [12] on droplet surfaces could give rise to saturation. Despite being a problem for most spectroscopic methods, saturation can often be compensated for by accepted and unproblematic precautions such as decreasing the path length. Mass spectrometers have no immediately obvious analogue of this experimental curative, so other factors have to be considered. Imaginative use of algorithmic correction has been reported [13], whereby an unsaturated peak in an isotope pattern is used to work backwards to a more accurate estimation of the abundance of that species. In cases where we are operating at concentrations at which saturation effects become troublesome, we deliberately detune the mass spectrometer. By adjusting various instrumental parameters, the analyte response can be improved and optimized [14]. The effect on the ion intensity can be greatly affected by the solvent of choice, the gas flow rate, and the source temperature settings of the instrument [15]. This work describes the warning signs of saturation and some of the parameters we change in order to obviate the effects of this phenomenon.

In this study, we primarily examined the trityl carbocation, $\left[\mathrm{Ph}_{3} \mathrm{C}\right]^{+}$, a reactive ionic compound used to abstract hydride or methide [16]. It is stable in the presence of a weakly coordinating anion (in this case, $\left.\left[\mathrm{B}\left(\mathrm{C}_{6} \mathrm{~F}_{5}\right)_{4}\right]^{-}\right)$, and as it is permanently positively charged, is easy to detect using electrospray ionization mass spectrometry (ESI-MS). But it is also reactive, so examining it at the micromolar concentrations typical of mass spectrometric analysis will usually result in decomposition. The study details ways in which we detuned the instrument such that higher concentrations that were largely free from decomposition could be studied without saturation effects playing a significant role. 


\section{Results and Discussion}

\section{Evidence for saturation}

Saturation can be indicated by various observations from a mass spectrum. The observations below demonstrate some examples of such, where the sample concentration is too high and the spectrum can no longer accurately characterizes the true concentration of an analyte.

\section{Peak shape}

For time-of-flight (TOF) mass analyzers, the peak shape is distorted most at the base of the peak. A peak shape that indicates saturation is illustrated in Figure 1. By simply zooming in a full-scale spectrum (1a) in the baseline, there is a distinct ramp before the peak, and a pronounced tailing afterwards (1b).

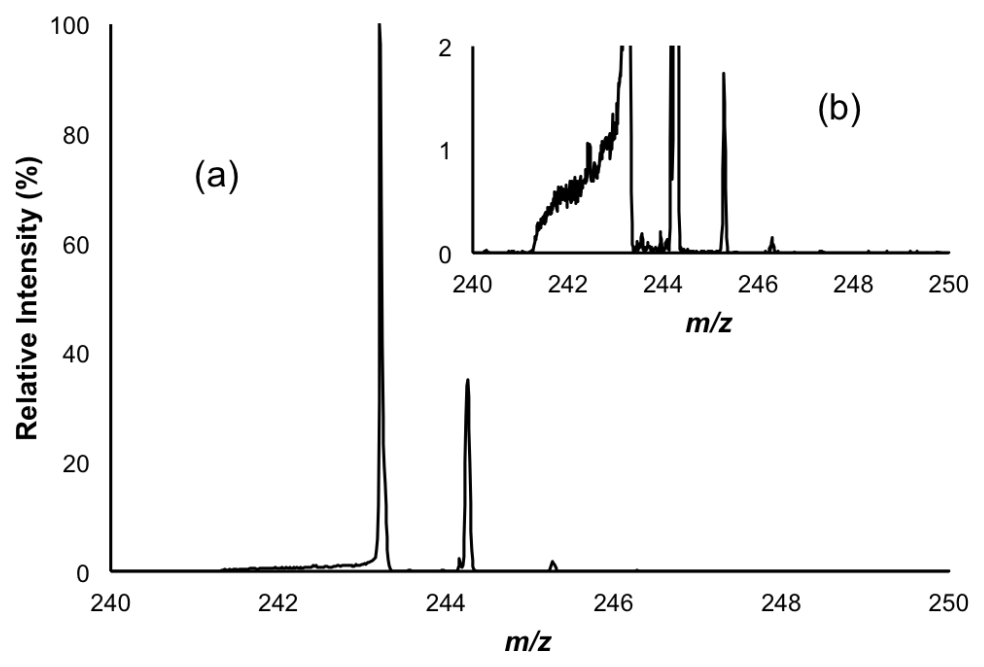

Figure 1. Peak shape of a saturated peak (a) full scale and (b) zoomed into the y axis by a factor of 20.

For triple quadrupole mass analyzers (TQD), the distortion is at the top of the peak. It gets truncated so that the peak shape is squared off. Tuning the resolution shows a significant effect; as the higher the resolution can be indicated by a sharper peak (Figure 2). By simply increasing the resolution of the mass analyzers, a saturation peak can be overcome and better resolution can be obtained. 
Figure 2. Peak shape of a

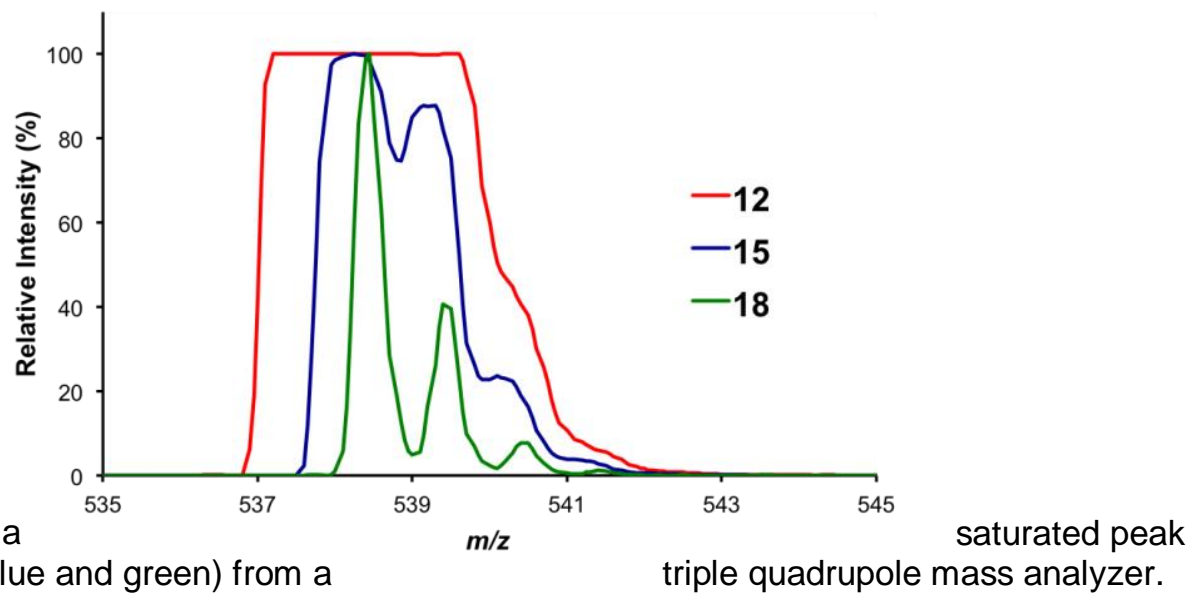

(red) and nonsaturated (blue and green) from a

triple quadrupole mass analyzer.

\section{Isotope pattern shape}

Saturation disproportionately affects the most intense peaks in the spectrum. Because isotope patterns contain peaks of different intensity, they are affected to different degrees. In practice, this means that the smaller peaks are larger than they should be, and the more intense peaks are less intense than anticipated. For hydrocarbon species, the ratio between the isotope pattern of $\mathrm{M}$, the mass of the most abundant isotope, and $\mathrm{M}+1$, should be $5: 1$ (Figure $3 \mathrm{~b}$ [17]). In this example, trityl cation, $\left[\mathrm{Ph}_{3} \mathrm{C}\right]^{+}$, has a less abundant peak at $\mathrm{m} / z 243$ and a more abundant isotope peak at $m / z 244$ (Figure 3a). The order of intensities, however, does not change.
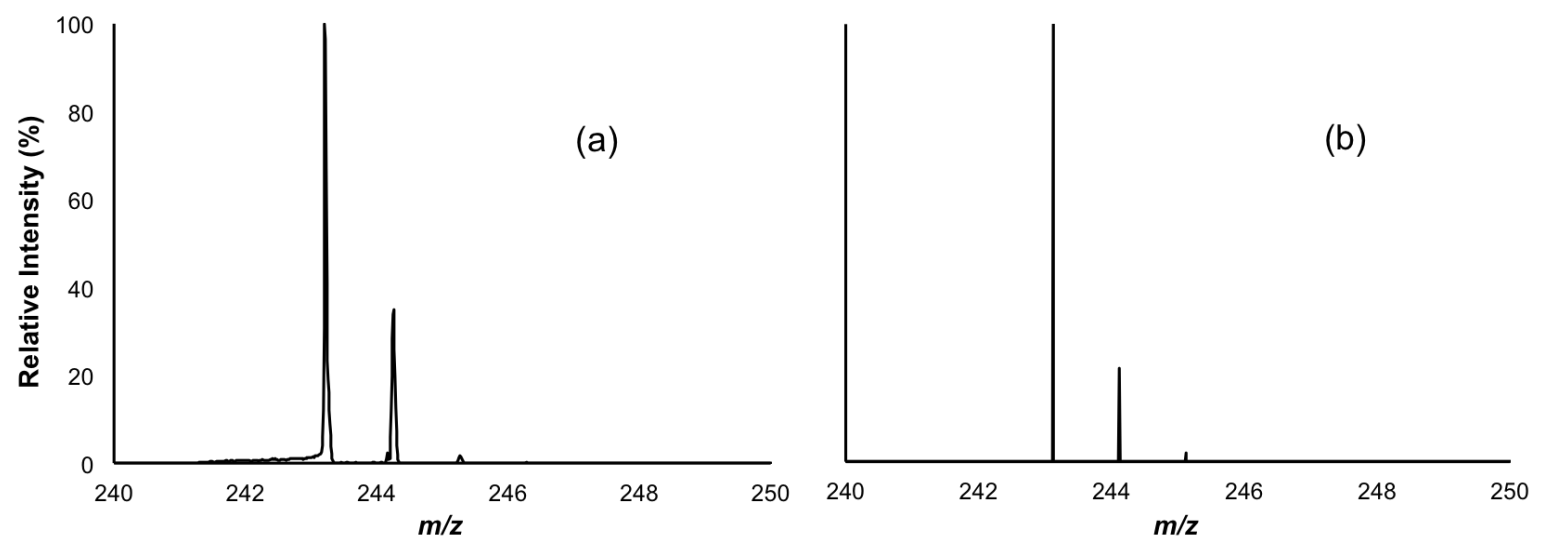

Figure 3. Isotope pattern shape of (a) saturated spectrum and (b) calculated spectrum of trityl cation $\left[\mathrm{Ph}_{3} \mathrm{C}\right]^{+}$.

The fact that the less abundant peaks are not saturated is a fact that can be exploited when tracking reactions as indicated and explained in details in the next section.

\section{Reaction traces}


Plotting reaction progress with a saturated peak gives data that is not just unreliable but nonphysical. It is most often characterized by a reactant that barely seems to diminish while a product appears to a large extent. As the reaction proceeds, the product itself may become saturated, so it climbs less than it should while the reactant diminishes normally. This behaviour can be observed in Figure 4a. Note in particular the crossover point between the two species: it should be at $50 \%$, but instead it is considerably above that value. In this particular example, two reactions are occurring, neutralization and isobutylation of trityl, and only the isobutylation product is observed, which is why both traces drop away to the baseline. A useful quick test for saturation involves looking at the traces for individual peaks in the isotopomer envelope. If no peaks are saturated, all of these traces should overlay once normalized (though the lower abundance peaks will necessarily generate a noisier trace). If the abundant peaks show divergent behaviour, saturation effects are likely in play. Figure 4(b) (for $M+1$ ) shows quite different behaviour to Figure 4(a), and is a much better representation of the actual solution behaviour. Figure 4(c) is for the $\mathrm{M}+2$ peak, which is low enough in intensity enough that the trace becomes rather noisy, but the trace is closer in behavior to 4(b) than to 4(a).
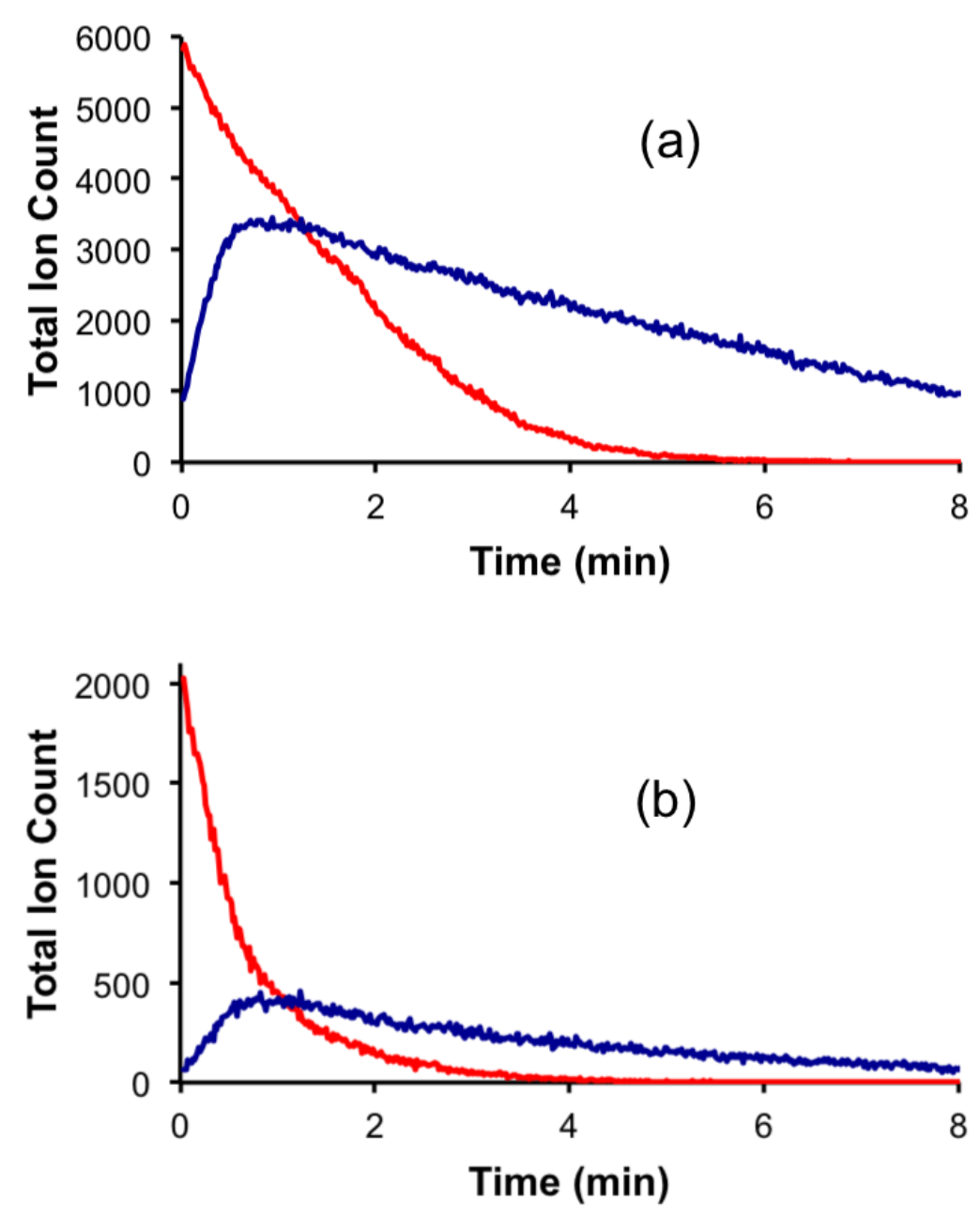


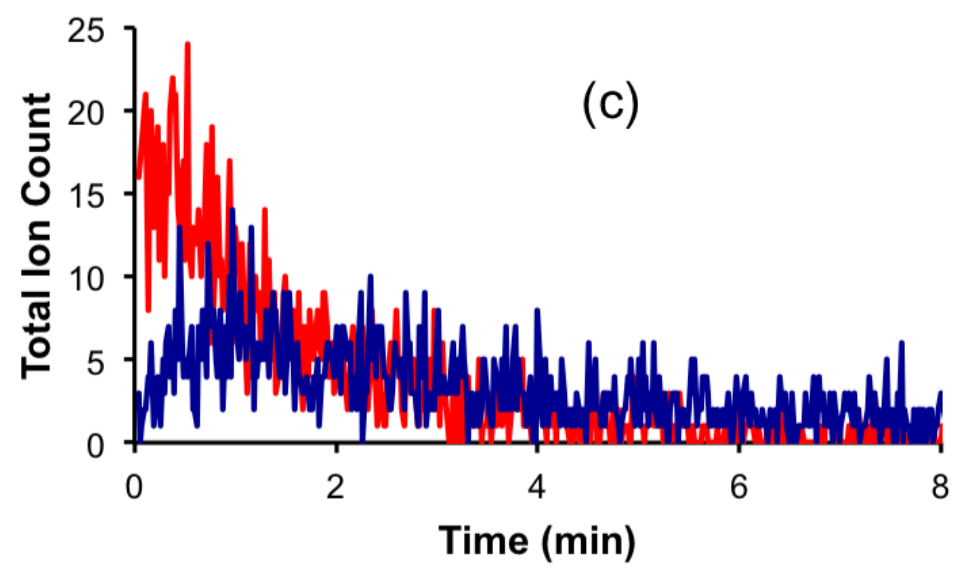

Figure 4. Reaction between $\left[\mathrm{Ph}_{3} \mathrm{C}\right]^{+}$and triisobutylaluminum (red) trityl peak $\left[\mathrm{Ph}_{3} \mathrm{C}\right]^{+}$and (blue) isobutylated trityl peak $\left[\mathrm{Ph}_{2} \mathrm{C}_{6} \mathrm{H}_{4} / \mathrm{Bu}\right]^{+}$. (a) $\mathrm{M}(\mathrm{m} / \mathrm{z} 243$ and 299) (b) $\mathrm{M}+1(\mathrm{~m} / \mathrm{z} 244$ and 300) (c) $\mathrm{M}+2(\mathrm{~m} / \mathrm{z}$ 245 and 301$)$.

Understanding saturation effects is crucial to correct interpretation of the abundance traces in a reaction [18][19]. There are experimental and data processing steps that can be taken to overcome the problem of saturation where dilution is not a practical solution, and these are outlined below.

\section{Mitigating Saturation}

There are various experimental strategies that can be attempted if saturation is a problem at high concentrations. Not all will be available on all instruments, and instruments other than the ones we have may very well have other options available. Here we report the instrumental parameters most effective for mitigating saturation in a quadrupole time-of-flight (Q-TOF) mass spectrometer.

Five parameters: the cone size, the microchannel plate detector voltage, the capillary voltage, the probe position, and the cone gas flow were examined. Three parameters were held constant while examining one particular parameter to allow comparisons. The optimal setting of each parameter is indicated by a linear calibration curve that corresponds to the increase of sample concentration. Curvature of the calibration response indicates saturation and more de-tuning has to be done.

\section{Detector sensitivity}

Saturation affects the detector in mass spectrometers: essentially, it reaches a point at which ions are arriving at the detector but are not registered. In orthogonal time-of-flight instruments, which use microchannel plate (MCP) detectors, saturation is straightforward to understand, because MCPs can only register one event in a given time window. So if at any given interval more than one ion arrival occurs, nonetheless only one arrival is registered. This is known as "digital dead time". Because the sensitivity of the MCP is dependent on the voltage applied to it [20], an obvious approach to reducing sensitivity is to lower that voltage. However, this approach has its own issues, particularly with thresholding, as seen in the figure below. At $2700 \mathrm{~V}$, saturation is clearly a problem, with curvature of the line being apparent very early in the trace. $2500 \mathrm{~V}$ is close to linear, but the lower voltage values do not start registering ions at all. 


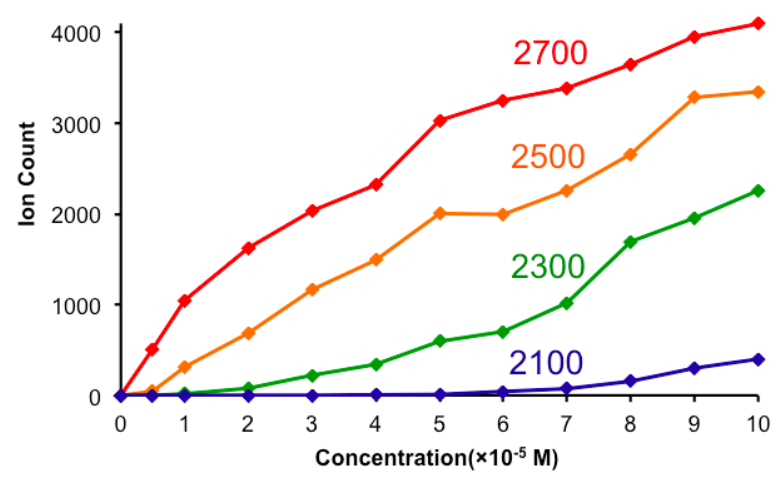

Figure 5. A graph of the calibration curves where the cone size $(0.36 \mathrm{~mm})$, the probe position (position 2), the cone gas flow (50 L/hour), and the capillary voltage (2700 V) were all held constant and only the MCP detector voltage changed.

\section{Capillary voltage}

Ionization source parameters are optimized to achieve better sensitivity of the instrument [21]. One of the main parameters of the ionization source, namely the capillary voltage applied to the spray capillary, serves multiple functions. It enables the electrospray plume to form [22], and also powers the underlying electrochemistry that generates the excess of positive ions needed for analysis [23]. Ion counts usually increase with capillary voltage, but there is a limited range available. The quality of the electrospray is highly dependent on the capillary voltage, as the formation of charged droplets is closely associated with a high voltage of the capillary [24]. Below a certain value, the electrospray plume will not form properly, and above a certain value, plasma discharges can form. Both of these manifest as highly erratic signals. Increasing the MCP detector voltage from $2000 \mathrm{~V}$ to $3000 \mathrm{~V}$ in increments of $250 \mathrm{~V}$ show that moderate values gives the most linear response (Figure 6); high values are prone to saturation and lower values show signs of thresholding effects, requiring a certain minimal concentration before any response at all is registered. 


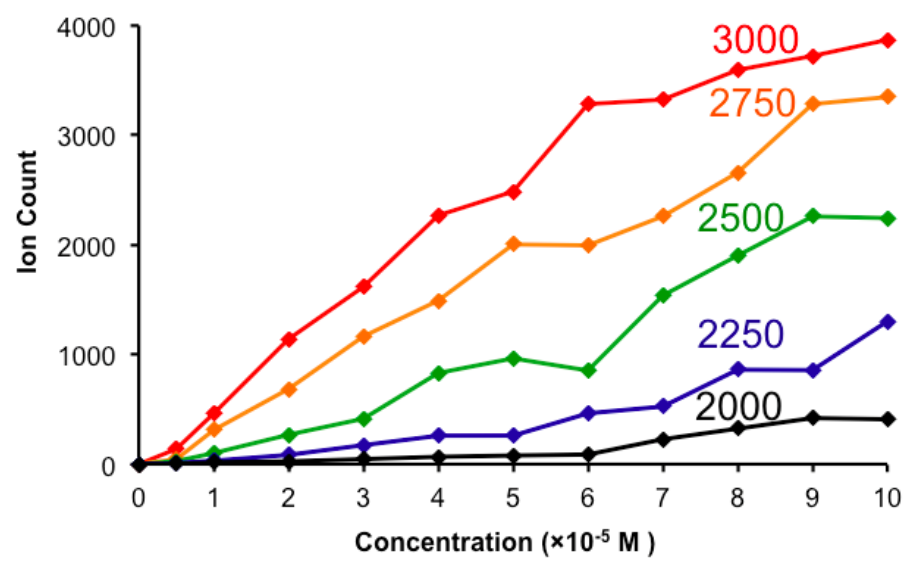

Figure 6. A graph of the calibration curves where the cone size $(0.36 \mathrm{~mm})$, the probe position (position $2)$, the MCP detector voltage (2500 V), and the cone gas flow (50 L/hour) were all held constant and only the capillary voltage changed.

\section{Probe position (capillary to cone distance)}

Some instruments allow the position of the capillary to be moved with respect to the orifice leading into the mass spectrometer. Moving this position can cause quite dramatic changes in signal intensity [25], and the relative abundance of ions can even change [26] (with smaller, more mobile ions losing less intensity with respect to their bulkier counterparts at greater distances). Five different capillary to cone distances were labelled and investigated (Figure 7) and the results were compared (Figure 8).

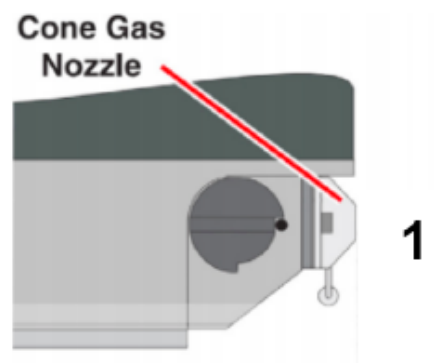


Figure 7. Probe positions (capillary tip to cone distance) investigated. Position $1(x=1 \mathrm{~mm}, y=3 \mathrm{~mm})$. Position $2(x=1 \mathrm{~mm}, y=6 \mathrm{~mm})$. Position $3(x=5 \mathrm{~mm}, y=4.5 \mathrm{~mm})$. Position $4(x=10 \mathrm{~mm}, y=3 \mathrm{~mm})$.

Position 5 ( $x=10 \mathrm{~mm}, y=6 \mathrm{~mm})$.

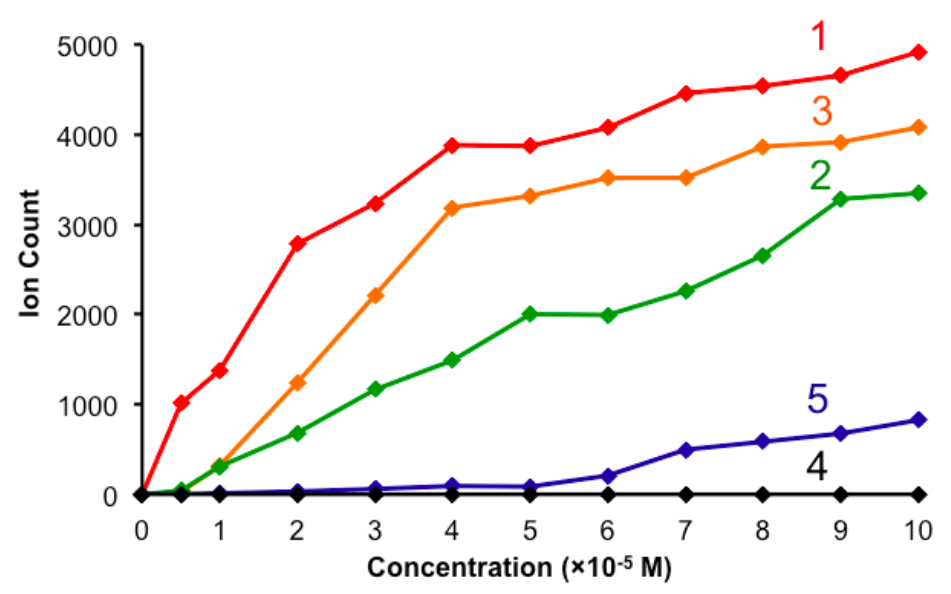

Figure 8. A graph of the calibration curves where the cone size $(0.36 \mathrm{~mm})$, the cone gas flow (50L/hour), the MCP detector voltage $(2500 \mathrm{~V})$, and the capillary voltage $(2700 \mathrm{~V})$ were all held constant and only the probe position changed.

The optimal probe position that indicates linear ion response according to the concentration of the analyte is the probe position 2. This probe position has therefore been held constant for acquiring the other experimental results in this paper. Position 4 provided no response at all, position 1 (and to a lesser extent 3) were saturation-prone and position 5 provided only a weak signal and then only at moderate concentrations.

\section{Cone gas flow}

There are two sources of source gases on the instrument, the desolvation gas, a coaxial flow emerging from around the capillary, and the cone gas, a flow emerging from the cone mounted at the orifice of the instrument. Increasing the cone gas has the effect of increasing desolvation but at highest values it can also make it harder for the ions to make their way from source to instrument [27] and can reduce the efficiency of ionization [28]. Adjusting the cone gas has similar effect to the MCP voltage, despite a dramatically different mechanism of action (Figure 9).

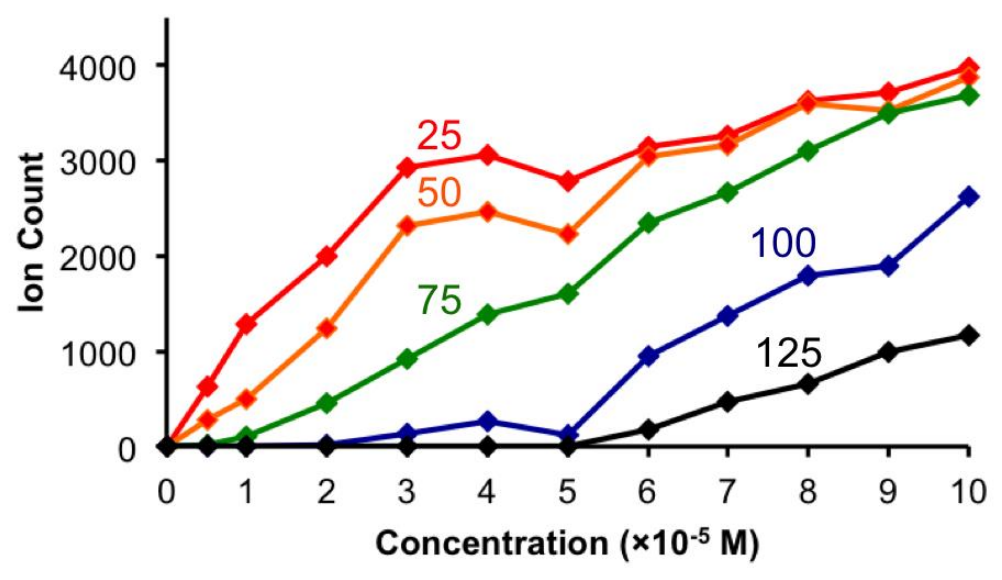


Figure 9. A graph where the calibration curves where the cone size $(0.36 \mathrm{~mm})$, the probe position (position 2), the MCP detector voltage $(2500 \mathrm{~V})$, and the capillary voltage $(2700 \mathrm{~V})$ were all held constant and only cone gas flow (L/hour) changed.

The results agreed with our hypothesis. Lowering the cone gas flow indeed shows a better desolvation, giving a much higher ion count but with the cost of saturation. With increments of 25 $\mathrm{L} /$ hour, we have found that in order to acquire the best linear curve, it is best to hold the cone gas flow at $75 \mathrm{~L} /$ hour for the solvent and concentration range considered here. This value is likely to be different for other solvents.

\section{Cone size}

The ion count as seen on the spectrum correlates to the number of ions entering from the sampling cone of the mass spectrometer. Tuning the amount of incoming ions is an effective way to avoid saturation as it affects the ion transmission efficiency [29] and detunes the sensitivity of the instrument. Two cones, with diameters of $0.80 \mathrm{~mm}$ and $0.36 \mathrm{~mm}$ were experimented with and the effect of the cone size was investigated (Figure 10).

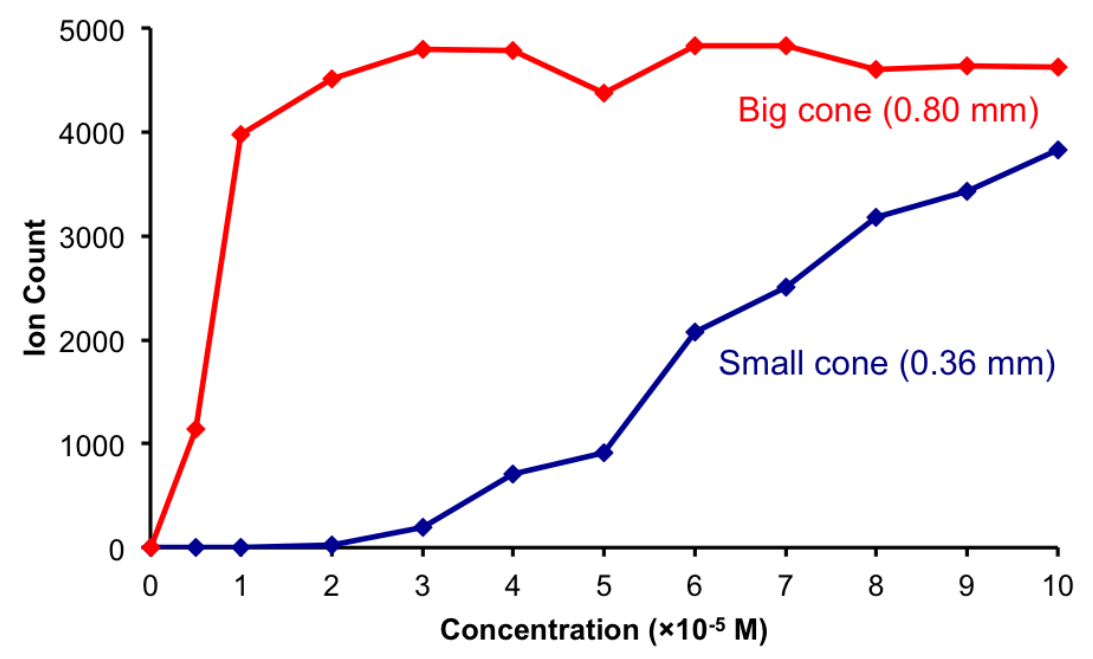

Figure 10. A graph where the calibration curves where the probe position (position 2), the MCP detector voltage $(2500 \mathrm{~V})$, the capillary voltage $(2700 \mathrm{~V})$, and the cone gas flow $(50 \mathrm{~L} / \mathrm{hour})$ all held constant and only the cone size changed.

Comparing the two cone sizes, our experimental results have shown the dramatic effect caused by the huge difference in the number of ions entering the mass spectrometer. A smaller cone size that effectively avoids the saturation issue is used for acquiring experimental results.

Besides de-tuning settings of a mass spectrometer to combat the saturation issue, recent instrumental developments have taken into the consideration of the saturation issue of mass spectrometer, as some modern instruments also sometimes possess settings that allow attenuation of the ion beam after the source but before encountering the mass analyzer(s). The Synapt G2Si, for example, has a setting called Continuum Dynamic Range Enhancement (DRE) 
[30] that introduces a baffle that diminishes the ion current making it into the mass spectrometer proper.

\section{Experimental}

Experiments were performed with a Waters Micromass Q-ToF Micro Mass Spectrometer. Trityl tetrakis(pentafluorophenyl)borate, $\left[\mathrm{Ph}_{3} \mathrm{C}\right]^{+}\left[\mathrm{B}\left(\mathrm{C}_{6} \mathrm{~F}_{5}\right)_{4}\right]^{-},\left(1.0 \mathrm{mg}, 1.0 \times 10^{-4} \mathrm{~mol}\right)$ was weighed out and fluorobenzene $(10 \mathrm{~mL})$ was added to prepare a solution with a concentration of $100 \mu \mathrm{M}(1.0$ $\times 10^{-4} \mathrm{M}$ ). Calibration curves were generated via the external calibration method. Twelve sample concentrations from 5 to $100 \mu \mathrm{M}\left(5.0 \times 10^{-6}\right.$ to $\left.1 \times 10^{-4}\right)$ were prepared upon further dilution. A sample was then injected via direct sample infusion with a $1 \mathrm{~mL}$ syringe and a syringe pump with a flow rate of $20 \mu \mathrm{L} / \mathrm{min}$. For each parameter adjusted, a different ESI-MS(+) spectrum was acquired for one minute where an average the total ion count at the ion peak $m / z 243 \mathrm{Da}$ was taken. A plot of ion intensity vs. concentration was generated.

\section{Conclusions}

When samples need to be more concentrated than is normal for mass spectrometric investigation, typically due to high reactivity, there are a range of strategies that can be employed to mitigate saturation effects. In this paper we reported the evidences of saturation in mass spectrometer and the strategies to overcome such limitations. With an optimal combination of the capillary voltage, the cone gas flow, the detector voltage, and capillary to cone distance, and the cone size, linear calibration curves that better reflect the true ion concentration can be generated. These encompass experimental detuning processes to deliberately lower sensitivity, and data processing that focuses on examining only those parts of the spectrum where saturation is not problematic. These tools offer researchers a variety of approaches when facing the difficult tradeoff between minimizing decomposition of their reactive sample vs. high concentrations causing saturation effects, with the caveat that the optimum values described here are likely to be different in other machines/solvents/analytes of interest.

\section{Acknowledgements}

J. S. M. thanks NSERC and NOVA Chemicals' Centre for Applied Research (Strategic Project Grant \# 478998-15) for operational funding and CFI, BCKDF, and the University of Victoria for infrastructural support. 


\section{References}

1. Fenn, J.B., Mann, M., Meng, C.K., Wong, S.F., Whitehouse, C.M.: Electrospray ionization for mass spectrometry of large biomolecules, (1989)

2. $\quad$ Zijlstra, H.S., Joshi, A., Linnolahti, M., Collins, S., Mclndoe, J.S.: Modifying methylalumoxane via alkyl exchange. Dalt. Trans. (2018). doi:10.1039/C8DT04242J

3. Yunker, L.P.E., Ahmadi, Z., Logan, J.R., Wu, W., Li, T., Martindale, A., Oliver, A.G., Mclndoe, J.S.: Real-Time Mass Spectrometric Investigations into the Mechanism of the Suzuki-Miyaura Reaction. Organometallics. (2018). doi:10.1021/acs.organomet.8b00705

4. Theron, R., Wu, Y., Yunker, L.P.E., Hesketh, A. V., Pernik, I., Weller, A.S., Mclndoe, J.S.: Simultaneous Orthogonal Methods for the Real-Time Analysis of Catalytic Reactions. ACS Catal. (2016). doi:10.1021/acscatal.6b01489

5. Zijlstra, H.S., Collins, S., Mclndoe, J.S.: Oxidation of Methylalumoxane Oligomers. Chem. - A Eur. J. (2018). doi:10.1002/chem.201705458

6. Yeung, D., Penafiel, J., Zijlstra, H.S., Mclndoe, J.S.: Oxidation of Titanocene(III): The Deceptive Simplicity of a Color Change. Inorg. Chem. (2018).

doi:10.1021/acs.inorgchem.7b02705

7. Zijlstra, H.S., Linnolahti, M., Collins, S., Mclndoe, J.S.: Additive and Aging Effects on Methylalumoxane Oligomers. Organometallics. (2017).

doi:10.1021/acs.organomet.7b00153

8. Alfaro, C.M., Uwakweh, A.O., Todd, D.A., Ehrmann, B.M., Cech, N.B.: Investigations of analyte-specific response saturation and dynamic range limitations in atmospheric pressure ionization mass spectrometry. Anal. Chem. (2014). doi:10.1021/ac502984a

9. Kebarle, P., Tang, L.: From ions in solution to ions in the gas phase - the mechanism of electrospray mass spectrometry. Anal. Chem. (1993). doi:10.1021/ac00070a001

10. Beach, D.G., Gabryelski, W.: Linear and nonlinear regimes of electrospray signal response in analysis of urine by electrospray ionization-high field asymmetric waveform ion mobility spectrometry-MS and implications for nontarget quantification. Anal. Chem. (2013). doi:10.1021/ac3027542

11. Enke, C.G.: A Predictive Model for Matrix and Analyte Effects in Electrospray lonization of Singly-Charged lonic Analytes. Anal. Chem. (1997). doi:10.1021/ac970095w

12. Zook, D.R., Bruins, A.P.: On cluster ions, ion transmission, and linear dynamic range limitations in electrospray (ionspray) mass spectrometry. Int. J. Mass Spectrom. Ion Process. (2002). doi:10.1016/s0168-1176(96)04511-9

13. Bilbao, A., Gibbons, B.C., Slysz, G.W., Crowell, K.L., Monroe, M.E., Ibrahim, Y.M., Smith, R.D., Payne, S.H., Baker, E.S.: An algorithm to correct saturated mass spectrometry ion abundances for enhanced quantitation and mass accuracy in omic studies. Int. J. Mass Spectrom. (2018). doi:10.1016/j.ijms.2017.11.003

14. Raji, M.A., Schug, K.A.: Chemometric study of the influence of instrumental parameters on ESI-MS analyte response using full factorial design. Int. J. Mass Spectrom. (2009). doi:10.1016/j.ijms.2008.10.013

15. Janusson, E., Hesketh, A. V., Bamford, K.L., Hatlelid, K., Higgins, R., Mclndoe, J.S.: Spatial effects on electrospray ionization response. Int. J. Mass Spectrom. (2015). doi:10.1016/j.ijms.2015.07.016

16. Bahr, S.R., Boudjouk, P.: Trityl tetrakis[3,5-bis(trifluoromethyl)phenyl]borate: a new hydride abstraction reagent. J. Org. Chem. (2005). doi:10.1021/jo00046a048

17. Patiny, L., Borel, A.: ChemCalc: A building block for tomorrow's chemical infrastructure. J. Chem. Inf. Model. (2013). doi:10.1021/ci300563h

18. Belli, R.G., Wu, Y., Ji, H., Joshi, A., Yunker, L.P.E., Mclndoe, J.S., Rosenberg, L.: Competitive Ligand Exchange and Dissociation in Ru Indenyl Complexes. Inorg. Chem. (2019). doi:10.1021/acs.inorgchem.8b02915 
19. Janusson, E., Zijlstra, H.S., Nguyen, P.P.T., Macgillivray, L., Martelino, J., Mclndoe, J.S.: Real-time analysis of $\mathrm{Pd} 2(\mathrm{dba}) 3$ activation by phosphine ligands. Chem. Commun. (2017). doi:10.1039/c6cc08824d

20. Dallüge, J., Vreuls, R.J.J., Beens, J., Brinkman, U.A.T.: Optimization and characterization of comprehensive two-dimensional gas chromatography with time-of-flight mass spectrometric detection (GC × GC - TOF MS). J. Sep. Sci. (2002). doi:10.1002/16159314(20020301)25:4<201::AID-JSSC201>3.0.CO;2-B

21. Kruve, A.: Influence of mobile phase, source parameters and source type on electrospray ionization efficiency in negative ion mode. J. Mass Spectrom. (2016).

doi:10.1002/jms.3790

22. Yamashita, M., Fenn, J.B.: Electrospray ion source. Another variation on the free-jet theme. J. Phys. Chem. (1984). doi:10.1021/j150664a002

23. Zhou, S., Cook, K.D.: A mechanistic study of electrospray mass spectrometry: Charge gradients within electrospray droplets and their influence on ion response. J. Am. Soc. Mass Spectrom. (2001). doi:10.1016/S1044-0305(00)00213-0

24. Ikonomou, M.G., Blades, A.T., Kebarle, P.: Electrospray-lon Spray: A Comparison of Mechanisms and Performance. Anal. Chem. (1991). doi:10.1021/ac00018a017

25. Benkestock, K., Sundqvist, G., Edlund, P.O., Roeraade, J.: Influence of droplet size, capillary-cone distance and selected instrumental parameters for the analysis of noncovalent protein-ligand complexes by nano-electrospray ionization mass spectrometry. J. Mass Spectrom. (2004). doi:10.1002/jms.685

26. Page, J.S., Marginean, I., Baker, E.S., Kelly, R.T., Tang, K., Smith, R.D.: Biases in Ion Transmission Through an Electrospray lonization-Mass Spectrometry Capillary Inlet. J. Am. Soc. Mass Spectrom. (2009). doi:10.1016/j.jasms.2009.08.018

27. Cai, S.S., Short, L.C., Syage, J.A., Potvin, M., Curtis, J.M.: Liquid chromatographyatmospheric pressure photoionization-mass spectrometry analysis of triacylglycerol lipidsEffects of mobile phases on sensitivity. J. Chromatogr. A. (2007). doi:10.1016/j.chroma.2007.10.008

28. Steenkamp, P.A., Harding, N.M., van Heerden, F.R., van Wyk, B.E.: Identification of atractyloside by LC-ESI-MS in alleged herbal poisonings. Forensic Sci. Int. (2006). doi:10.1016/j.forsciint.2005.11.010

29. Wilm, M., Mann, M.: Analytical properties of the nanoelectrospray ion source. Anal. Chem. (1996). doi:10.1021/ac9509519

30. Cryar, A., Groves, K., Quaglia, M.: Online Hydrogen-Deuterium Exchange Traveling Wave Ion Mobility Mass Spectrometry (HDX-IM-MS): a Systematic Evaluation. J. Am. Soc. Mass Spectrom. (2017). doi:10.1007/s13361-017-1633-z 